Check for updates

Cite this: Mater. Adv., 2022, 3,1687

Received 26th July 2021. Accepted 1st January 2022 DOI: 10.1039/d1ma00644d

rsc.li/materials-advances

\title{
Wood-based composite materials for ultralight lens antennas in 6G systems $\dagger$
}

\author{
Mikko Kokkonen, (D) *a Mikko Nelo, (D) ${ }^{a}$ Henrikki Liimatainen, (D) ${ }^{b}$ Jonne Ukkola, (D) ${ }^{b}$ \\ Nuutti Tervo, (D) ${ }^{c}$ Sami Myllymäki, (D) ${ }^{a}$ Jari Juuti (D) and Heli Jantunen (D) ${ }^{a}$
}

\begin{abstract}
Extremely high frequencies used in future wireless communication systems such as 6G require low loss materials to avoid wasting power and maintain acceptable efficiency. Furthermore, especially in the internet of things (IOT) applications, low weight and the possibility to focus the radiation pattern in the desired direction would also improve the communication between units and reduce the required signal power. Radio frequency (RF) lenses for signal focusing can be made from low loss and low relative permittivity materials. In this work, the development of sustainable composites with a relative permittivity of $\sim 1.15$ and loss tangents in the range of $10^{-3}$ is presented. The composites were fabricated at the exceptionally low temperature of $95^{\circ} \mathrm{C}$ and were based on hollow micron-sized glass spheres and three different types of cellulose nanofibers as the water-soluble binder. A simple manufacturing method through casting and drying is presented. The surface properties of the composites were investigated with surface profile analysis and the dielectric properties by SPDR and terahertz spectroscopy. The weight of the fabricated lens was $0.6 \mathrm{~g}$ and the transmittance was $99.85 \%$. The lens improved the antenna gain by $14-18 \mathrm{dBi}$ depending on the surface smoothening. Also, coating the lens with a moisture protecting agent did not alter the lens performance.
\end{abstract}

\section{Introduction}

The frequencies used in wireless communication systems are constantly increasing and will soon reach the $\mathrm{THz}$ band (0.1-1 THz). ${ }^{1}$ At these high frequencies, new, low relative permittivity $\left(\varepsilon_{r}\right)$, very low dielectric loss $(\tan \delta)$, lightweight and sustainable materials are needed. One of the radio frequency (RF) components that benefits from new materials is the RF lens used as in optics to focus the radiation thus increasing the antenna gain. ${ }^{2}$ RF lenses are commonly made from materials such as high resistivity silicon $\left(\varepsilon_{r} \sim 11.9\right)^{3-5}$ and polymers $\left(\varepsilon_{r} \sim 2.3\right) .^{3,6-8}$ The use of materials with low $\varepsilon_{r}$ and low $\tan \delta$ allows higher signal speeds and improved efficiency. ${ }^{9}$ However, polymers and silicon are heavy materials that in turn increase the relative weight of the lens compared to the highly integrated RF electronics. There is a need to develop lightweight and sustainable materials for RF use.

In order to further reduce the relative permittivity of the polymeric materials, both structural porosity ${ }^{10-12}$ and low

\footnotetext{
${ }^{a}$ Microelectronics Research Unit, University of Oulu, FI-90014, Oulu, P.O. Box 4500, Finland. E-mail: Mikko.Kokkonen@oulu.fi

${ }^{b}$ Fiber and Particle Engineering, University of Oulu, FI-90014, Oulu, P.O. Box 4300, Finland

${ }^{c}$ Center for Wireless Communications, University of Oulu, FI-90014, Oulu, P.O. Box 8000, Finland

$\dagger$ Electronic supplementary information (ESI) available. See DOI: 10.1039/d1ma00644d
}

permittivity functional groups ${ }^{13-15}$ have been employed. Utilization of porosity has resulted in permittivity values as low as 1.05 with excellent dielectric losses in the polyethylene and polyurethane foams used in radomes. ${ }^{16,17}$ On the other hand, hollow glass microspheres (HGMS) offer an inexpensive solution to reduce the dielectric permittivity and losses of circuit boards, ${ }^{18}$ thick film devices ${ }^{19}$ or EM-shielding. ${ }^{20,21}$ Recently, they have been used to produce ultra-low permittivity substrate materials ${ }^{22}$ and $\mathrm{RF}$ lenses. ${ }^{23}$ In addition to this, cellulose nanofiber (CNF) suspension can be used as a biobased binder just by mixing it with filler materials without the use of additional pressure or a strong shearing force. This enables the use of HGMS as a porosity-adding material without the risk of crushing the microspheres during mixing and further processing steps. Typically extrudable materials are thermoplastic polymers, which require high $>160{ }^{\circ} \mathrm{C}$ temperatures. In this work, one of the aims was to develop a suspension which could be extruded in room temperature.

In this work, the RF lenses were made by casting different CNF-HGMS suspensions into molds. The combination of CNFs and HGMS could form a composite, which is a lightweight, non-plastic, sustainable, and environmentally friendly composite. Dielectric values $\left(\varepsilon_{r}, \tan \delta\right)$ of the samples were measured with the split post dielectric resonator method (SPDR) at 2.5 and $5.2 \mathrm{GHz}$. The dielectric properties in the $\mathrm{THz}$ band (0.1-1 THz) were measured with terahertz spectroscopy. 
The performance of the RF lenses was evaluated with a lens measurement system (at $300 \mathrm{GHz}$ ). Their surface quality was analyzed with a laser scanning microscope. Also, the feasibility of different nanocellulose materials, i.e. cellulose nanofibers possessing inherent cellulose structure without any additional functional groups, TEMPO oxidized CNF containing carboxylates, and wood nanofibers (WNF) having a lignin containing surface, fabricated in this work was compared against two common lens materials, silicon and Teflon.

\section{Experimental}

\section{A. Materials and chemicals}

HGMS were purchased from Kevra Oy (Finland). According to the manufacturer's datasheet the spheres were C-type glass with $50-75 \% \mathrm{SiO}_{2}$ and had an average particle size of $40-80 \mu \mathrm{m}$, a volumetric density of $0.1-0.15 \mathrm{~g} \mathrm{~cm}^{-3}$ and a temperature resistance of up to $650{ }^{\circ} \mathrm{C}$. According to the field emission scanning electronic microscope with energy dispersive spectroscopy (FESEM-EDS) analysis carried out in the previous work, ${ }^{22}$ the HGMS contained $72 \mathrm{wt} \% \mathrm{SiO}_{2}, 14 \mathrm{wt} \% \mathrm{Na}_{2} \mathrm{O}$ and $14 \mathrm{wt} \%$ CaO. 2,2,6,6-Tetramethylpiperidinyl-1-oxy radical (TEMPO) and choline chloride $(>98.0 \%)$ were both obtained from TCI (Germany). Urea $\left(\mathrm{NH}_{2} \mathrm{CONH}_{2} ;>99.0 \%\right)$, sodium bromide $(\mathrm{NaBr})$, and lithium chloride ( $\mathrm{LiCl})$ were ordered from Sigma-Aldrich (Finland).

\section{B. Preparation of three different types of cellulose nanofibers}

Three different types of cellulose nanofibers (CNF) were utilized. The CNF was obtained from either deep eutectic solvent (DES) treatment (DES CNF), TEMPO oxidized CNF (TO-CNF) or wood nanofiber (WNF). In brief, the DES CNF had an inherent cellulose structure without any additional functional groups, ${ }^{24}$ TO-CNF contained carboxylates, ${ }^{25}$ while WNF had a lignin containing surface and had a surface charge density of close to zero. $^{26}$

DES-treated CNF was synthesized from bleached birch-wood pulp (Betula Pendula) using a non-derivatizing DES pretreatment based on urea-choline chloride. ${ }^{24}$ First, the DES system was formed by heating $324 \mathrm{~g}$ choline chloride and $244.5 \mathrm{~g}$ urea in a round-bottom flask at $100{ }^{\circ} \mathrm{C}$ in an oil bath to obtain a clear, colorless liquid, after which the cellulose pulp (5.65 $\mathrm{g}$ of ripped small pieces of dry pulp sheets) was introduced into the liquid at $100{ }^{\circ} \mathrm{C}$ over 2 hours. After the DES pretreatment, $500 \mathrm{ml}$ of deionized water was added while mixing and the cellulose fibers were filtered (Whatman 413) and washed with deionized water $(2000 \mathrm{ml})$. The pretreated sample with a concentration $(0.5 \% \mathrm{w} / \mathrm{w})$ was then passed five times through $400 \mu \mathrm{m}$ and $200 \mu \mathrm{m}$ diameter chambers at a pressure of 1000 bar using a microfluidizer (Microfluidics M-110EH-30, USA). The obtained viscous cellulose nanofiber suspension was stored in a cold environment $\left(+4{ }^{\circ} \mathrm{C}\right)$.

Anionic TO-CNF was prepared according to a previously reported method. ${ }^{27} 10 \mathrm{~g}$ of bleached birch pulp was suspended in water $(1000 \mathrm{ml})$ containing TEMPO $(0.16 \mathrm{~g}, 0.1 \mathrm{mmol})$ and sodium bromide ( $1 \mathrm{~g}, 1 \mathrm{mmol}) .10 .0 \mathrm{mmol}$ of $\mathrm{NaClO}$ solution was added as drops to the cellulose suspension, and the mixture was stirred at room temperature and kept at $\mathrm{pH} 10$ for $4 \mathrm{~h}$. The oxidation was stopped by adding $10 \mathrm{ml}$ of ethanol, and the oxidized fibers were completely washed by filtration with deionized water. The oxidized cellulose fiber suspension with a solid content of $1 \% \mathrm{w} / \mathrm{w}$ was passed through a pair of chambers $(400 \mu \mathrm{m}$ and $200 \mu \mathrm{m}$ diameter) under a pressure of 1000 bar using a microfluidizer (Microfluidics M-110EH-30, USA) to obtain TEMPO-oxidized cellulose nanofibers (TO-CNF).

WNF was produced using a previously reported procedure with slight modifications. ${ }^{26}$ In short, unbleached spruce groundwood pulp $(1.5 \% \mathrm{w} / \mathrm{w})$ was ground using a Masuko Super MassColloider (MKCA6-2 J CE; Masuko Sangyo, Japan) at $\sim 90{ }^{\circ} \mathrm{C}$. The distance between the grinding stones was gradually decreased while the pulp was repeatedly fed through the grinder to obtain WNF.

Chemical characteristics of CNF samples were determined using Diffuse reflectance infrared Fourier transform (DRIFT) spectroscopy. For WNF, the lignin content was measured with a The TAPPI T 222 om-02 (GWP27.4 wt\%, BP 0.2 wt\%); the acetone-soluble extractives with a TAPPI T 280 pm-99 (GWP2.2 wt\%, BP 0.1 wt\%); and the hemicellulose and degraded cellulose with a TAPPI T 212 om-02 (GWP $13.0 \mathrm{wt} \%$, BP $3.9 \mathrm{wt} \%$ ). The charge density of CNFs were revealed by the electric conductivity titration method using a method described by Rattaz et al. ${ }^{28}$ The diameter of nanocelluloses were analyzed from transmission electron microscope (TEM) images of $>100$ individual nanoparticles. The properties of CNF samples have been summarized in Table 1.

\section{Preparation of the CNF/HGMS lenses}

Mixtures of different CNF suspensions and HGMS were made by adding the HGMS powder into CNF suspensions until the suspension was thick enough to barely pass through the nozzle of a $2 \mathrm{ml}$ syringe. Due to the similar water contents of CNF suspension and HGMS, this proved to be roughly $50: 50$ by volume, i.e., 2.0 grams of HGMS and $12.0 \mathrm{~g}$ of CNF suspension.

The quantity of CNF in suspension was as follows: TO-CNF = $1 \mathrm{wt} \%$, DES CNF $=1.96 \mathrm{wt} \%, \mathrm{WNF}=2.5 \mathrm{wt} \%$. As the viscosity of each CNF suspension was similar, they were used in similar volumetric ratios with HGMS to achieve similar extrudability. resulting the TO-CNF/HGMS suspension to contain $0.6 \mathrm{vol} \%$ dried CNF, DES CNF/HGMS suspension 1.2 vol\% dried CNF and WNF/HGMS suspension 1.5 vol\% dried CNF. Volume of the CNF in suspensions was calculated on the basis of estimated density of $1.63 \mathrm{~g} \mathrm{~cm}^{-3}$ by Aulin et al. ${ }^{29}$

Table 1 Characteristics of CNF samples

Anionic surface charge Average diameter

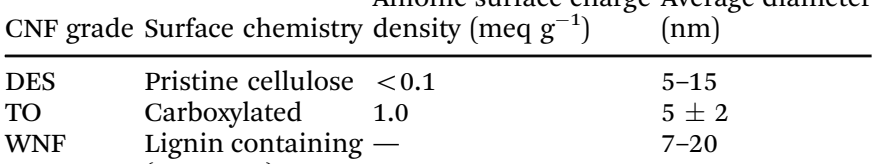

(27.4 wt\%) 


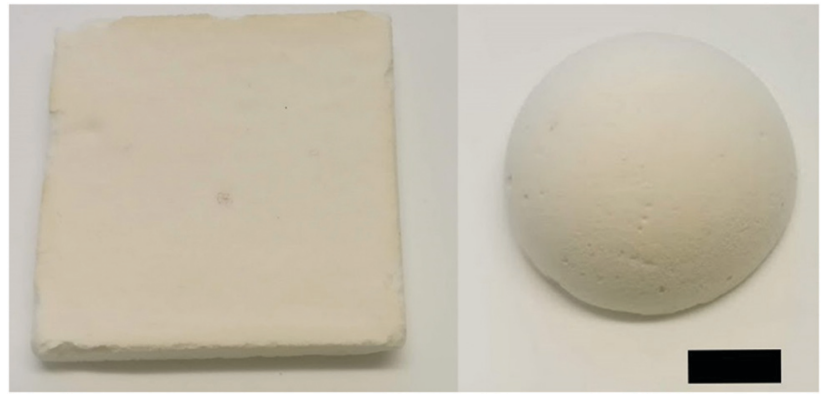

Fig. 1 Example of square and lens shaped samples. Binder: WNF. Scale bar: $1 \mathrm{~cm}$.

This also resulted in relatively small shrinkage of the resulting samples. Samples for 2.5 and $5.2 \mathrm{GHz}$ SPDR measurements were made by applying the HGMS/CNF paste into a $2 \mathrm{~mm}$ thick, $60 \mathrm{~mm}$ diameter mold. Samples were dried in the mold overnight at $95{ }^{\circ} \mathrm{C}$, removed and kept for 2 hours at $105^{\circ} \mathrm{C}$. Samples for higher frequency measurements and for the RF lenses were made by applying the HGMS/CNF pastes into silicone molds made into the desired shape, either a $30 \mathrm{~mm}$ hemisphere or a $35 \times 35 \times 5 \mathrm{~mm}$ square. The samples were dried at room temperature for 24 hours, followed by 16 hours at $95{ }^{\circ} \mathrm{C}$ and $2 \mathrm{~h}$ at $105{ }^{\circ} \mathrm{C}$. Examples of the resulting samples are shown in Fig. 1. An ultralight weight $(\sim 0.6 \mathrm{~g})$ was measured for the lens.

The lenses had tiny holes which formed during the drying process because of trapped air bubbles. The surface roughness was reduced by filling these pores with a thin layer of HGMSCNF paste made with TEMPO -oxidized CNF and HGMS with 1:1 volumetric proportion. The coated and dried surface was finally manually smoothed with 2000-grit sandpaper while keeping the original shape of the lens.

\section{Dielectric and surface measurements}

The dielectric measurements at 2.5 and $5.2 \mathrm{GHz}$ were carried out with a vector network analyzer (VNA) (Rhodes \& Schwarz ZVB20, Germany) with SPDR (QWED, Poland) at $2.475 \mathrm{GHz}$ and $5.180 \mathrm{GHz}$. The measurement results were calculated using QWED analysis software developed for the resonators. The dielectric measurements for $0.1-1 \mathrm{THz}$ were conducted with terahertz spectroscopy (TeraPulse 4000 (teraview, UK)). The surface of the lenses was measured with a 3D laser scanning microscope (Keyence VK-X200, USA).

\section{Results and discussion}

\section{A. Dielectric measurements for low and high frequency}

The measured dielectric values at 2.5 and $5.2 \mathrm{GHz}$ are shown in Table 2. All the composite samples had very similar relative permittivity values (in the range of $1.14 \pm 0.01$ ) at both frequencies, indicating that the surface chemistry or charge density of CNFs had only a minor effect on performance of lenses (Table 1). Presumably, the most important parameter was the dimensions of CNFs, which were revealed to be similar with all samples. The loss tangents of the TEMPO-type CNF
Table 2 Dielectric properties at 2.5 and $5.2 \mathrm{GHz}$

\begin{tabular}{|c|c|c|c|c|}
\hline \multirow[b]{2}{*}{ Binding material } & \multicolumn{2}{|l|}{$2.5 \mathrm{GHz}$} & \multicolumn{2}{|l|}{$5.2 \mathrm{GHz}$} \\
\hline & $\varepsilon_{\mathrm{r}} \pm 0.01$ & $\tan \delta \pm 2 \mathrm{E}-5$ & $\varepsilon_{\mathrm{r}} \pm 0.01$ & $\tan \delta \pm 2 \mathrm{E}-5$ \\
\hline \multicolumn{5}{|c|}{ Permittivity of nanocelluloce HGMS composite } \\
\hline DES CNF & 1.14 & 0.0026 & 1.14 & 0.0026 \\
\hline WNF & 1.15 & 0.0026 & 1.15 & 0.0025 \\
\hline TO-CNF & 1.13 & 0.0019 & 1.13 & 0.0019 \\
\hline \multicolumn{5}{|c|}{ Permittivity of cellulose nanofibers as dried film } \\
\hline DES CNF & 3.73 & 0.1 & 3.63 & 0.1 \\
\hline WNF & 3.85 & 0.1 & 3.78 & 0.1 \\
\hline TO-CNF & 4.93 & 0.1 & 4.72 & 0.1 \\
\hline
\end{tabular}

binder were lower than others, possibly due to the lower CNF content. The CNF content of suspensions was determined by gravimetric method, i.e. evaporating the CNF film dry and measuring the weight difference.

Terahertz time-domain spectroscopy (THz-DTS) was used to measure the real and imaginary parts of the $\varepsilon_{r}$ for the higher frequency (Fig. 2). The DES CNF sample had slightly higher permittivity than the TO-CNF and WNF, whereas the imaginary values were close to each others. At $600 \mathrm{GHz}$ and above the resonance effect from the glass spheres was seen in the higher permittivity. The permittivity values (Table 3) at $150 \mathrm{GHz}$ were 1.16 for DES CNF, 1.15 for WNF and 1.15 for TO-CNF, and for $300 \mathrm{GHz}$ values were 1.15 for DES CNF, 1.15 for WNF and 1.15 for TO-CNF. $\tan \delta$ values were between $0.002-0.003$ at $150 \mathrm{GHz}$ and slightly higher (0.003-0.004) at $30 \mathrm{GHz}$. These loss values are sufficient for RF applications.

The permittivity of the individual CNFs ranges from 3 to 5 and they have a high losses. HGMS had permittivity of $\sim 1.13$ and losses $\sim 0.004$ making it an excelent component to lower the permittivity and losses of the proposed composite.

\section{B. Evaluating the lens performance through the $S_{21}$}

The lens measurement system is shown in Fig. 3. The setup consisted of a VNA (PNA-X $68 \mathrm{GHz}$ Keysight, USA), and a pair of frequency extenders (220-330 GHz, Virginia diodes, USA) which had either a waveguide (transmitter side) or horn antenna (receiver side) connected. The lens was positioned at $90 \mathrm{~mm}$ distance from the transmitter, at the point with the highest $\mathrm{S}_{21}$ $\mathrm{dB}$ reading. The measurement was conducted by moving the lens in $y$ - and $z$ - directions to create a $20 \times 20$ grid where each point represented $1 \mathrm{~mm}$ of movement together with the recorded received signal $\left(S_{21}\right)$. The lens was moved by using MATLAB controlled positioners based on stepper motors.

Overall, six measurements were conducted and the results at $300 \mathrm{GHz}$ are presented in Fig. 4. Each ring represents $1 \mathrm{~dB}$ transition, and the darkest red ring represents the highest measured value. Also, to have comparable results, the maximum $\mathrm{dB}$ value from Fig. 4 a was used as a $0 \mathrm{~dB}$ reference point for Fig. 4b-f. Five lenses were measured which were as follows:

Two lenses smoothed and unsmoothed (kept at room humidity) to measure the effect of the lens smoothing.

Two lenses smoothed and unsmoothed (kept 1.5 days in a humidity chamber $(85 \% \mathrm{RH}))$ to measure the effect from long 

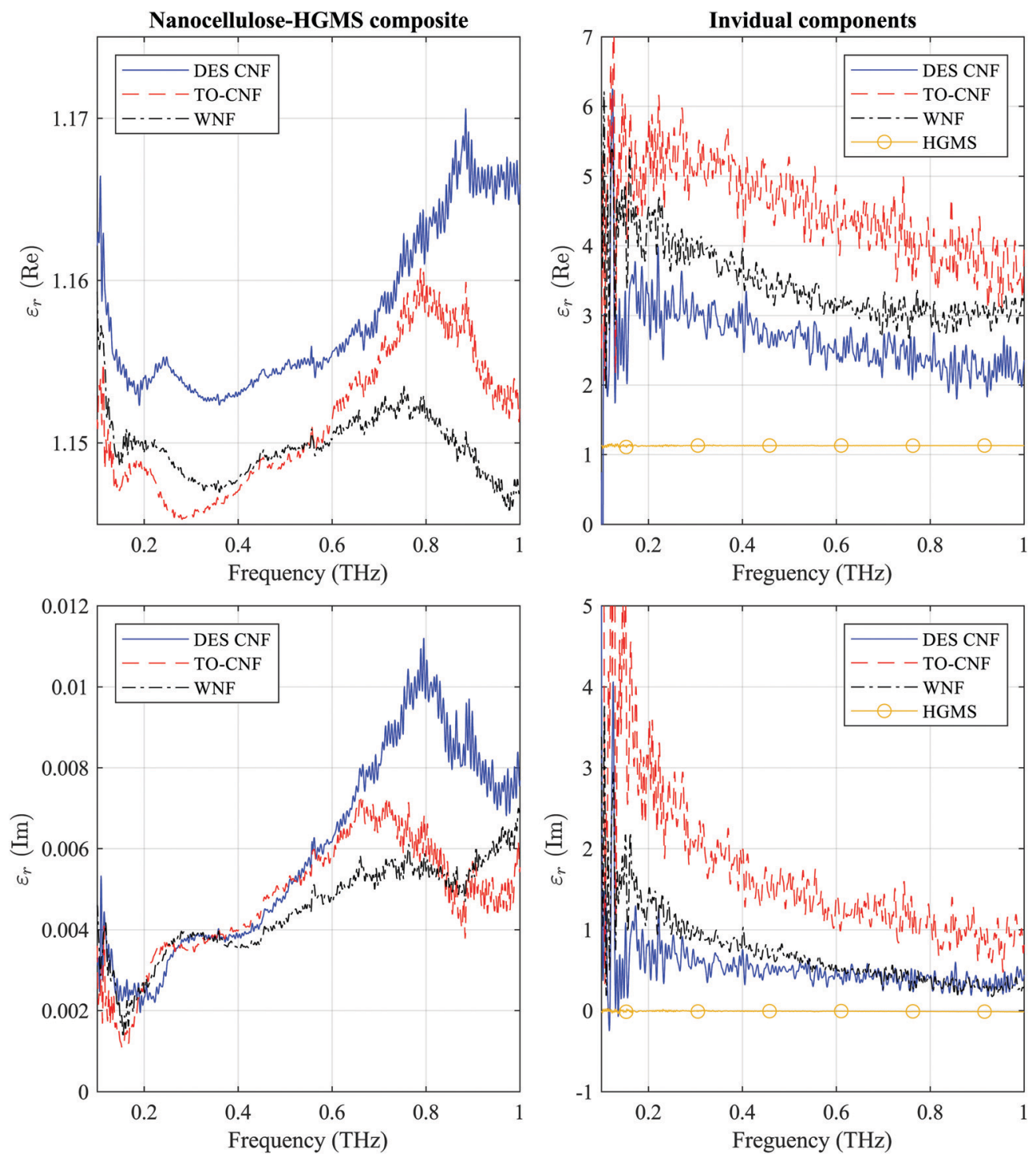

Fig. 2 (at left) Dielectric real (Re) and imaginary (Im) values of different types of cellulose nanofibers (CNF) hollow glass microspheres (HGMS) composites. (at right) Invidual components (CNFs measured from thin film $<100 \mu \mathrm{m}$ thickness).

Table 3 Dielectric properties at 150 and $300 \mathrm{GHz}$

\begin{tabular}{|c|c|c|c|c|c|c|}
\hline \multirow[b]{2}{*}{ Binding material } & \multicolumn{3}{|c|}{$150 \mathrm{GHz}$} & \multicolumn{3}{|c|}{$300 \mathrm{GHz}$} \\
\hline & $\begin{array}{l}\operatorname{Re} \varepsilon_{\mathrm{r}} \pm \\
0.01\end{array}$ & $\begin{array}{l}\operatorname{Im} \varepsilon_{\mathrm{r}} \pm \\
0.001\end{array}$ & $\begin{array}{l}\tan \delta \pm \\
0.01\end{array}$ & $\begin{array}{l}\operatorname{Re} \varepsilon_{\mathrm{r}} \pm \\
0.01\end{array}$ & $\begin{array}{l}\operatorname{Im} \varepsilon_{\mathrm{r}} \pm \\
0.001\end{array}$ & $\begin{array}{l}\tan \delta \pm \\
0.01\end{array}$ \\
\hline \multicolumn{7}{|c|}{ Permittivity of nanocelluloce HGMS composite } \\
\hline DES CNF & 1.16 & 0.003 & 0.003 & 1.15 & 0.004 & 0.004 \\
\hline WNF & 1.15 & 0.002 & 0.002 & 1.15 & 0.004 & 0.004 \\
\hline TO-CNF & 1.15 & 0.002 & 0.002 & 1.15 & 0.004 & 0.003 \\
\hline \multicolumn{7}{|c|}{ Permittivity of invidual components } \\
\hline DES CNF & 3.59 & 0.544 & 0.152 & 2.97 & 0.88 & 0.296 \\
\hline WNF & 5.09 & 2.15 & 0.422 & 4.00 & 0.96 & 0.240 \\
\hline TO-CNF & 6.34 & 5.56 & 0.877 & 5.14 & 2.13 & 0.414 \\
\hline HGMS & 1.13 & -0.005 & 0.004 & 1.13 & -0.003 & 0.003 \\
\hline
\end{tabular}

term moisture exposure on the unsmoothed and smoothed lenses.

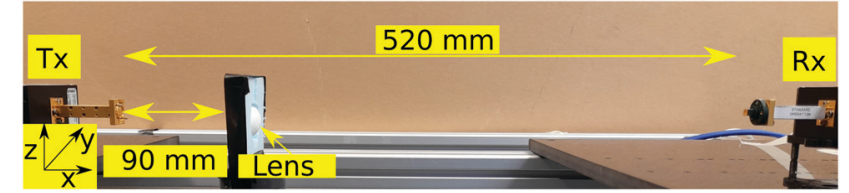

Fig. 3 Measurement setup to measure electric field deflected by the lens. The lens is inside a foam holder which is supported by a rod which can be moved in $x^{-}, y^{-}, z$-directions by stepper motors between the transmitter $(T x)$ and receiver $(R x)$. As the lens is the only moving part, the recorded intensity of the $S_{21}$ does not have false readings (i.e., intensity changes) caused by cable movements.

One unsmoothed lens which was coated with a moisture protective layer $\left(\right.$ glaco $\left.^{30}\right)$. The lens was measured with and without the coating to study its effect on the lens performance. 

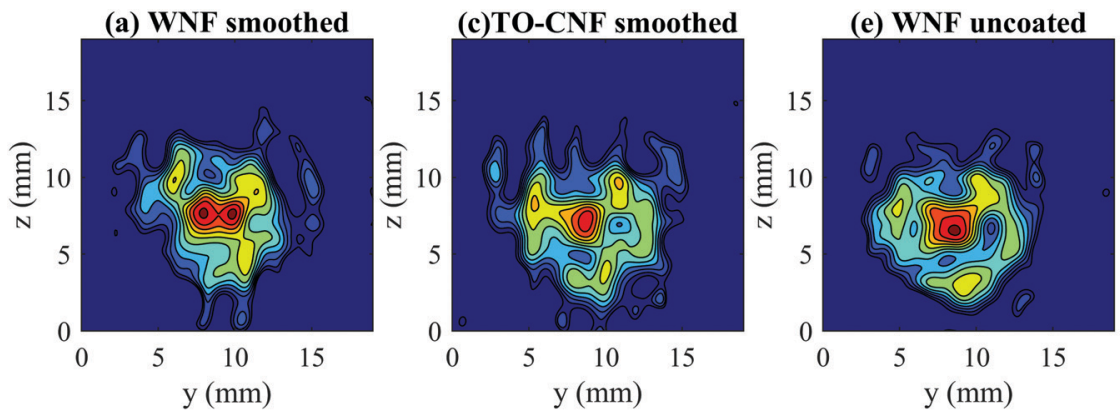

\section{(b) WNF unsmoothed}

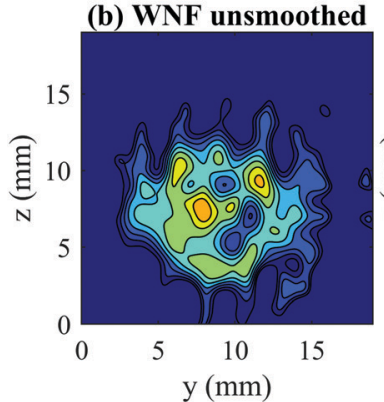

(d) TO-CNF unsmoothed
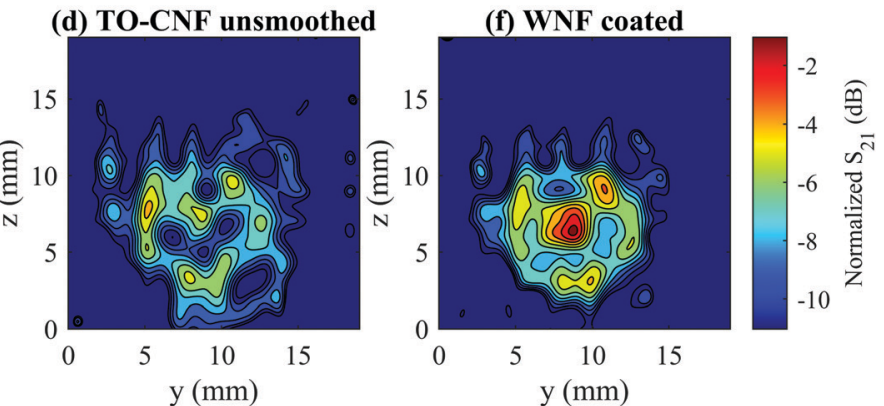

Fig. 4 Measured $S_{21}$ response from fabricated lenses at $300 \mathrm{GHz}$. (a) Smoothed and (b) unsmoothed WNF type lenses which were kept at room humidity ( 25\% RH). (c) Smoothed and (d) unsmoothed TO-CNF type lenses which were kept in humidity chamber (1.5 days, $85 \%$ RH). (e) Uncoated and (f) coated WNF type lens; lens was coated with a moisture protection (glaco ${ }^{30}$ ).

The results showed that without smoothing (Fig. 4(b and d)) the lens did not have a clear focus spot at the center but rather a ununiform "splash" of high and low dB areas. With smoothing (Fig. 4(a and c)), the focus spot formed to the center of the "splash". Improvement from the smoothing was $\sim 4 \mathrm{~dB}$.

To study the effect of humidity on the performance of the lenses the following experiments were performed. Two lenses were kept in the humidity chamber (85\% RH, 1.5 days) to allow as much moisture as possible to accumulate into the lens. The lenses were removed from the humidity chamber, installed to the measurement setup, and the measurement was conducted in minutes. The accumulated moisture was expected to reduce the performance of the lens by many dB (Fig. 4(c and d)). However, this effect was not observed, and the maximum intensity of the focal spot remained similar to that of the lens which was kept in room humidity comparing the smoothed lenses (Fig. 4(a and c)). This mean that the moisture exposure did not alter the performance of the proposed lens antenna at $300 \mathrm{GHz}$, despite the cellulose nanomaterials may have promoted the moisture adsorption of lenses.

As the electronics are typically protected from the moisture, the effect from the moisture protecting agent was also measured. The moisture protection agent glaco ${ }^{30}$ was used and there were no indications that the lens performance was either degraded or improved due to the addition of the moisture protection layer (Fig. 4(e) and (f)). Also, waterproofing efficiency of the coating layer was investigated by dropping water droplets on to the lens. It was noted that without the protective layer, the droplets were absorbed into the lens but with the coating the droplets were observed to roll off the lens surface (there is a supplementary video of this droplet experiment).

\section{Simulated radiation pattern}

Simulations were conducted with a CST microwave studio, ${ }^{31}$ lens distance to the waveguide (WR3.4) was $90 \mathrm{~mm}$ as in the measurements. The measured dielectric parameters such as $1.15 \varepsilon_{\mathrm{r}}$ and $0.0035 \tan \delta$ at $300 \mathrm{GHz}$ were used for the simulated lens.

Radiation patterns of the waveguide and the proposed lens antenna are shown in Fig. 5. The waveguide has gain of $\sim 7 \mathrm{dBi}$ and wide main lobe. With the lens the main lobe has been narrowed to 1.5-degree and the gain was $\sim 25 \mathrm{dBi}$ and side lobe level (SLL) of $\sim 12 \mathrm{~dB}$. Similar gain improvement $(\sim+18 \mathrm{dBi})$ was observed in the measurements with the smoothed lens.

\section{Roughness of the lens surface}

Topography was used to study the surface quality of the lenses. The surface images were taken from the tip of the smoothed,

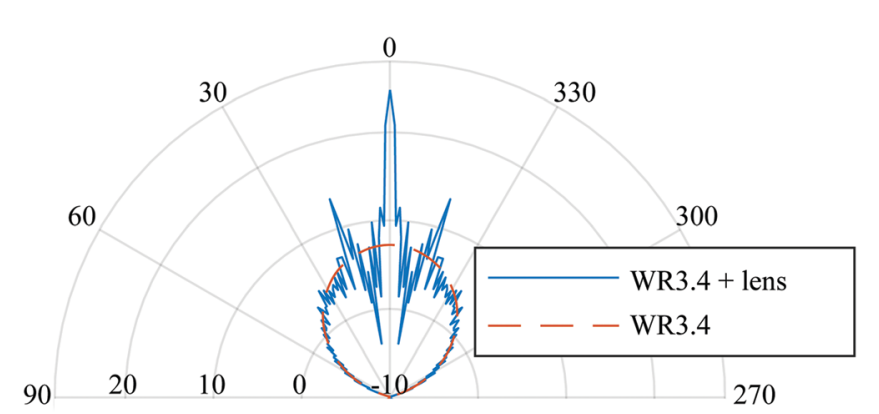

Fig. 5 Simulated radiation patterns of WR3.4 waveguide and proposed lens antenna at $300 \mathrm{GHz}$. Scale is in $\mathrm{dBi}$. 


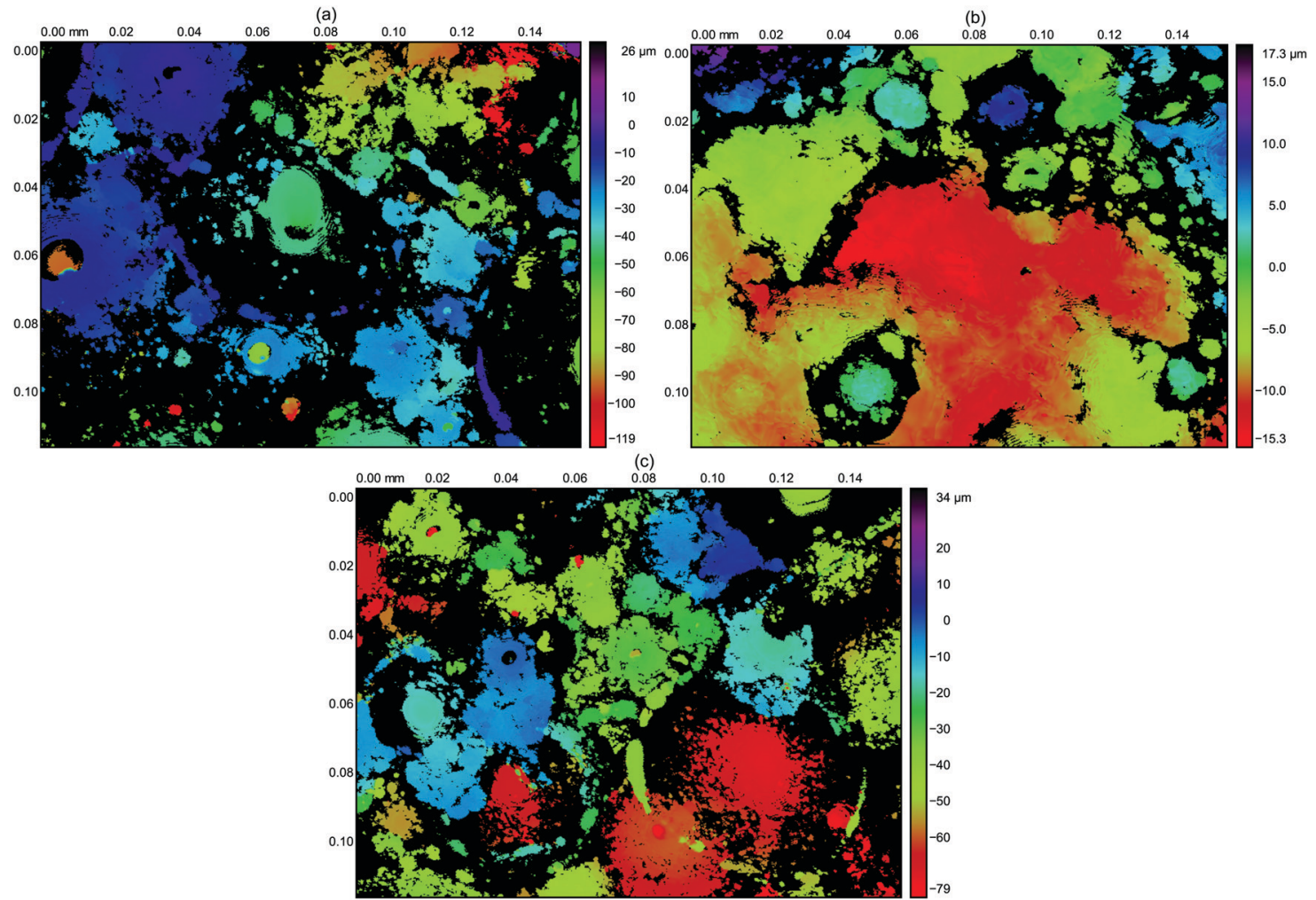

Fig. 6 Surface topography images of the (a) unsmoothed (b) smoothed and (c) coated lens. Without the smoothing lens surface has a large height deviation i.e., large Ra value.

unsmoothed, and coated lenses (Fig. 6). The unsmoothed lens (Fig. 6(a)) had a very rough surface ( $\mathrm{Ra} 29.6 \pm 6.5 \mu \mathrm{m})$ which explained its degraded RF performance. The Fig. 6(b) showed that the smoothing improved the $R_{\mathrm{a}}$ value to $8.8 \pm 1.6 \mu \mathrm{m}$. The coated lens had an Ra of $35.3 \pm 6.6 \mu \mathrm{m}$, which was close to the value measured with the nonsmoothed lens, and indicated that the coating layer was spread uniformly to some extent and did not act as a smoothing agent.

\section{E. Comparison of the wood composite lenses with other commonly used lenses}

A radio lens can be made from almost any material if its properties are suitable for the radio use. As the most common materials are silicon and plastic materials (Teflon), Table 4 shows their electrical properties at $300 \mathrm{GHz}$ against the nanocellulose used in this work. The calculated weight of silicon and Teflon lenses were quite similar and relatively high when compared to the nanocellulose ones. Thus, by using the nanocellulose the weight of the lens can be reduced by $~ 97 \%$ making it ultralight. This can potentially reduce the overall weight of the transceivers, especially in highly integrated sensor devices and drones.

As the materials have different loss tangent and permittivity, the power loss in the material was simulated. As the wave travels through the lens, some of the wave power is lost based on the following equation, ${ }^{32}$

$$
P=\pi f \tan (\delta) \varepsilon_{0} \varepsilon_{r} \int|\vec{E}|^{2} \delta V .
$$

Basically, the power loss $P$ in the material depends on the frequency $f$ of the traveling wave, the permittivity $\varepsilon_{r}$ and the loss tangent $\tan \delta$ of the material and the on the square of the absolute value of the electric field $|\vec{E}|^{2}$ inside the volume of the lens $V$. As the nanocellulose composite has such a high loss tangent it experiences a similar power loss in the material as silicon $(\sim 0.02 \%)$.

The signal not only travels inside the lens but also needs to enter and exit the lens as well. For entering and exiting, physical properties such as reflectance and transmittance become relevant. Since the silicon lens has a high permittivity, $30 \%$ of the exiting or entering signal becomes lost (I.e., it is reflected from the lens surface). To reduce this reflection loss, an anti-reflection coating can be used (I.e., impedance matching) and the physical requirement for such a layer is as follows; it has to be of a certain thickness, typically odd multiple of $\frac{\lambda}{4}$, and the permittivity of the antireflection layer should be $\sqrt{\varepsilon_{r}}$ related to the previous layer permittivity. ${ }^{33}$

To have a smooth transition to the air, single or multiple anti-reflection layers might be needed which increases the complexity of the lens structure, makes fabrication more difficult and ultimately raises the cost of the lens. With low permittivity lenses there is no need to use a matching layer to reduce the back reflection which makes the lens easier to manufacture and cheaper. It is also worthwhile to note that the nanocellulose lenses show very high total transmittance ( 99.9\%). 
Table 4 Comparison of nanocellulose lenses to common lens materials at $300 \mathrm{GHz}$

\begin{tabular}{llllllll}
\hline Material & Density $\mathrm{g} \mathrm{cm}^{-3}$ & Weight $^{b} \mathrm{~g}$ & \multicolumn{1}{c}{$\varepsilon_{\mathrm{r}}$} & $\tan \delta, \mathrm{E}-3$ & Power loss in material $^{c} \%$ & Reflectance \% & Total transmittance \% \\
\hline Silicon & 2.33 & 16.5 & 11.90 & 0.2 & 0.024 & 30.31 & 3.36 \\
Teflon & 2.22 & 15.6 & 2.10 & 0.2 & 0.005 & 0.12 & 96.67 \\
DES CNF $^{a}$ & 0.08 & 0.6 & 1.15 & 3.5 & 0.023 & 99.85 \\
WNF $^{a}$ & 0.08 & 0.6 & 1.15 & 3.5 & 0.023 & 0.12 & 99.85 \\
TO-CNF $^{a}$ & 0.08 & 0.6 & 1.15 & 3.5 & 0.023 & 0.12 & 99.85
\end{tabular}

${ }^{a}$ Different CNF/HGMS composite made in this work. ${ }^{b}$ Calculated for $30 \mathrm{~mm}$ half spherical lens. ${ }^{c}$ Simulated via CST microwave studio.

\section{Conclusion and perspectives}

A method with only three processing steps for manufacturing extremely low $\varepsilon_{r}$ and low $\tan \delta$ composite materials and RF lenses at very low temperature is presented. The sustainable composite with TEMPO-type CNF binder (TO-CNF) exhibited $\varepsilon_{r}$ and $\tan \delta$ values of 1.13 and 0.0019 at $5 \mathrm{GHz}$, respectively. At $300 \mathrm{GHz} \varepsilon_{r}$ was slightly higher (1.15) and $\tan \delta$ slightly lower (0.0017). The simulated power loss in the material was $0.023 \%$ which could be improved even further in the future by reducing the dielectric losses with a lower alkali content of HGMS.

The proposed method is also environmentally friendly due to its low energy consumption achieved by the low fabrication temperature of $95{ }^{\circ} \mathrm{C}$. Moreover, since only a low fabrication temperature and pressure are used, no special devices with high investment costs are required. The manufactured lenses were also ultralight $(0.6 \mathrm{~g})$ and high transmittance (>99\%). Ultralight lens antennas for 6G systems could improve antenna gain by $14-18 \mathrm{dBi}$ depending on the smoothing level of the lens surface.

In future, new sustainable and environmentally friendly materials should be developed for radio technology.

\section{Conflicts of interest}

There are no conflicts to declare.

\section{Acknowledgements}

This work was supported partly by Academy of Finland 6Genesis Flagship (Grant no. 318927) and partly by European Regional Development Fund project: Novel digitally fabricated materials for electronics, optics and medical applications (NOVIDAM).

\section{References}

1 M. Latva-aho and K. Leppänen ed., Key drivers and research challenges for $6 G$ ubiquitous wireless intelligence, Oulun Yliopisto, Oulu, 2019, http://urn.fi/urn:isbn:9789526223544 (accessed: 29 November 2021).

2 J. Volakis, Antenna Engineering Handbook, McGraw-Hill, 4th ed., 2007.

3 R. Sauleau, C. A. Fernandes and J. R. Costa, 11th International Symposium on Antenna Technology and Applied Electromagnetics [ANTEM 2005], St. Malo, 2005, pp. 1-5.
4 A. J. Alazemi, H. Yang and G. M. Rebeiz, Double Bow-Tie Slot Antennas for Wideband Millimeter-Wave and Terahertz Applications, IEEE Trans. Terahertz Sci. Technol., 2016, 6, 682-689.

5 J. Grzyb, M. Andree, R. Jain, B. Heinemann and U. R. Pfeiffer, A Lens-Coupled On-Chip Antenna for Dual-Polarization SiGe HBT THz Direct Detector, IEEE Antennas Wirel. Propag. Lett., 2019, 18, 2404-2408.

6 E. Lacombe, F. Gianesello, A. Bisognin, E. Lacombe, C. Luxey, A. Bisognin, D. Titz, H. Gulan, T. Zwick, J. Costa and C. A. Fernandes, in 2017 IEEE International Symposium on Antennas and Propagation USNC/URSI National Radio Science Meeting, San Diego, CA, 2017, pp. 5-6.

7 N. Chudpooti, N. Duangrit, P. Akkaraekthalin, I. D. Robertson and N. Somjit, 220-320 GHz Hemispherical Lens Antennas Using Digital Light Processed Photopolymers, IEEE Access, 2019, 7, 12283-12290.

8 S. Myllymäki, M. Teirikangas and M. Kokkonen, BaSrTiO3 ceramic-polymer composite material lens antennas at 220$330 \mathrm{GHz}$ telecommunication applications, Electron. Lett., 2020, 56, 1165-1167.

9 R. Farrell, T. Goshal, U. Cvelbar, N. Petkov and M. A. Morris, Advances in Ultra Low Dielectric Constant Ordered Porous Materials, Electrochem. Soc. Interface, 2011, 20, 39.

10 J. S. Rathore, L. V. Interrante and G. Dubois, Ultra Low-k Films Derived from Hyperbranched Polycarbosilanes (HBPCS), Adv. Funct. Mater., 2008, 18, 4022-4028.

11 A. M. Joseph, B. Nagendra, K. P. Surendran and E. Bhoje Gowd, Syndiotactic Polystyrene/Hybrid Silica Spheres of POSS Siloxane Composites Exhibiting Ultralow Dielectric Constant, ACS Appl. Mater. Interfaces, 2015, 7, 19474-19483.

12 B. Zhao, C. Zhao, C. Wang and C. B. Park, Poly(vinylidene fluoride) foams: a promising low- $\mathrm{k}$ dielectric and heatinsulating material, J. Mater. Chem. C, 2018, 6, 3065-3073.

13 C. Yuan, K. Jin, K. Li, S. Diao, J. Tong and Q. Fang, Non-Porous Low- $k$ Dielectric Films Based on a New Structural Amorphous Fluoropolymer, Adv. Mater., 2013, 25, 4875-4878.

14 Y. Liu, C. Qian, L. Qu, Y. Wu, Y. Zhang, X. Wu, B. Zou, W. Chen, Z. Chen, Z. Chi, S. Liu, X. Chen and J. Xu, A Bulk Dielectric Polymer Film with Intrinsic Ultralow Dielectric Constant and Outstanding Comprehensive Properties, Chem. Mater., 2015, 27, 6543-6549.

15 X. Lei, M. Qiao, L. Tian, Y. Chen and Q. Zhang, Tunable Permittivity in High-Performance Hyperbranched Polyimide 
Films by Adjusting Backbone Rigidity, J. Phys. Chem. C, 2016, 120, 2548-2561.

16 P. P. Eccostock | Laird Performance Materials, https://www. laird.com/sites/default/files/2021-01/RFP-DSPP\%2006242020.pdf, (accessed 3 March 2021).

17 Dielectric Foam Material - RF-2200 Dielectric Polyurethane Foam, https://www.generalplastics.com/wp-content/uploads/ 2017/09/RF-2200-PDS.pdf, (accessed 3 March 2021).

18 L. N. Chellis, R. M. Japp, W. J. Summa, W. J. Rudik and D. W. Wang, US5126192A, 1992.

19 D. Kellerman, CA pat., CA1331914C, 1994.

20 W. Fu, S. Liu, W. Fan, H. Yang, X. Pang, J. Xu and G. Zou, Hollow glass microspheres coated with $\mathrm{CoFe}_{2} \mathrm{O}_{4}$ and its microwave absorption property, J. Magn. Magn. Mater., 2007, 316, 54-58.

21 J. Wang, J. Wang, B. Zhang, Y. Sun, W. Chen and T. Wang, Combined use of lightweight magnetic $\mathrm{Fe}_{3} \mathrm{O}_{4}$-coated hollow glass spheres and electrically conductive reduced graphene oxide in an epoxy matrix for microwave absorption, J. Magn. Magn. Mater., 2016, 401, 209-216.

22 M. Nelo, H. Liimatainen, M. Väätäjä, J. Ukkola, J. Juuti and H. Jantunen, Solid Air-Low Temperature Manufacturing of Ultra-Low Permittivity Composite Materials for Future Telecommunication Systems, Front. Mater., 2019, 6, 94.

23 M. Kokkonen, M. Nelo, J. Chen, S. Myllymäki and H. Jantunen, Low Permittivity Environmentally Friendly Lenses for Ku Band, Prog. Electromagn. Res. Lett., 2020, 93, $1-7$.
24 J. Antti Sirviö, M. Visanko and H. Liimatainen, Deep eutectic solvent system based on choline chloride-urea as a pretreatment for nanofibrillation of wood cellulose, Green Chem., 2015, 17, 3401-3406.

$25 \mathrm{~K}$. Zhang and H. Liimatainen, Hierarchical Assembly of Nanocellulose-Based Filaments by Interfacial Complexation, Small, 2018, 14, 1801937.

26 M. Visanko, J. A. Sirviö, P. Piltonen, R. Sliz, H. Liimatainen and M. Illikainen, Mechanical fabrication of high-strength and redispersible wood nanofibers from unbleached groundwood pulp, Cellulose, 2017, 24, 4173-4187.

27 T. Saito, S. Kimura, Y. Nishiyama and A. Isogai, Cellulose Nanofibers Prepared by TEMPO-Mediated Oxidation of Native Cellulose, Biomacromolecules, 2007, 8, 2485-2491.

28 A. Rattaz, S. P. Mishra, B. Chabot and C. Daneault, Cellulose nanofibres by sonocatalysed-TEMPO-oxidation, Cellulose, 2011, 18, 585.

29 C. Aulin, M. Gällstedt and T. Lindström, Oxygen and oil barrier properties of microfibrillated cellulose films and coatings, Cellulose, 2010, 17, 559-574.

30 Glaco Mirror Coat Zero | Products | Glaco Windshield Coating Agent and Wiper Series, http:/www.glaco.jp/english/products/ mirror/glaco_mirrorcoat_0.html, (accessed 4 March 2021).

31 CST Studio Suite 3D EM simulation and analysis software, https:/www.3ds.com/products-services/simulia/products/ cst-studio-suite/, (accessed 25 August 2021).

32 CST Studio Suite 3D EM simulation user guide 2019. 33 B. D. Guenther, Modern optics, John Wiley, 1990. 